\title{
Tissue Models and Speckle Reduction in Medical Ultrasound Images
}

\author{
Radim Kolář and Jiří Jan \\ Department of Biomedical Engineering, FEEC, \\ Brno University of Technology, Czech Republic \\ kolarr@feec.vutbr.cz
}

\begin{abstract}
This paper presents a new method for speckle noise reduction in medical ultrasound images. It is based on the statistical description of the envelope of ultrasound signal by the virtue of the Nakagami-m distribution. Parameter of this distribution is used to adjust an adaptive filter.
\end{abstract}

\section{Introduction}

Ultrasound tissue modeling can provide an important information that can be used for diagnosis, image segmentation, interpretation or visualization. There are many tissue models based on various kind of probability distribution describing tissue from more or less complex way - Rayleigh or Rice distribution [4, K-distribution [3] or Nakagami distribution. We will focus on the Nakagami distribution 7, 8 that is able to distinguish between various kind of tissue conditions.

The aim of this paper is to describe a statistical based approach to ultrasound image processing (Section 2) and to utilize Nakagami distribution (Section 3) for speckle suppression (Section 44). Therefore, the speckles are considered as a noise makes the manual/automatic image segmentation difficult. Section 5 presents some results and discussion.

\section{$2 \quad$ Echo Model}

The echo signal can be considered as a sum of backscattered and backreflected single echoes from a number of scattering points and strong reflectors in the tissue [3. We can express this echo signal (in one point/time) with the help of phasor notation. Each scatter reflects $x_{k}$ amount of signal with the phase shift $\theta_{k}$ (due to the random location)

$$
X=\sum_{i=0}^{N-1} x_{i} \cdot e^{j \theta_{i}}
$$


In most cases the amplitude $x_{i}$ can be considered deterministic and we can rewrite (1) as

$$
X=\frac{1}{\sqrt{N}} \sum_{i=0}^{N-1} \alpha_{i} \cdot e^{j \theta_{i}}
$$

where $\alpha_{i}=\frac{x_{i}}{\sqrt{N}}$ are normalized to $\sqrt{N}$. This normalization means that the scattered amplitudes from each echo is equal. Mean values, $\mathrm{E}\{$.$\} , of the real and$ imaginary parts can be considered as a zero [3]: $E\left\{X_{r}\right\}=E\left\{X_{i}\right\}=0$. This holds for independent and identical distributions for $\alpha_{i}$ and $\theta_{i}$. For this condition these mean values are zero, because $\theta_{i}$ is assumed to be equally distributed within the interval $[-\pi, \pi]$. For the second moments, it can be shown that:

$$
E\left\{X_{i}\right\}=E\left\{X_{r}\right\}=\sigma^{2}
$$

Now, we express the radiofrequency signal as a time signal by involving $\omega_{0}$ :

$$
s(t)=X_{r}(t) \cdot \cos \left(\omega_{0} t\right)+j \cdot X_{i}(t) \cdot \sin \left(\omega_{0} t\right) .
$$

With the clinical scanner we can obtain only the inphase (the first) component. For envelope detection we assume the analytical nature of this signal and the quadrature (the second) component is obtain by the virtue of the Hilbert transform $(H T)$. The envelope is simply obtained by

$$
S(t)=\sqrt{X_{r}^{2}(t)+X_{i}^{2}(t)}=\sqrt{X_{r}^{2}(t)+H T\left\{X_{r}(t)\right\}^{2}} .
$$

\section{$3 \quad$ Nakagami Distribution}

We would like to describe various tissues conditions using only one universal mathematical model. This may be done using the Nakagami distribution. The probability density function of the envelope, $f(S)$, under the Nakagami model is given by [7]

$$
f(S)=\frac{2 m^{m} S^{2 m-1}}{\Gamma(m) \Omega^{m}} \cdot e^{-\frac{m}{\Omega} S^{2}}
$$

where $\mathrm{m}$ is the Nakagami parameter and $\Omega$ is the scaling parameter. The SNR value for Nakagami distributed envelope is given by:

$$
S N R_{N}=\frac{\text { mean }}{\text { standard deviation }}=\frac{E[S]}{\sqrt{E\left[S^{2}\right]-E[S]^{2}}}=\frac{1}{\sqrt{m\left[\frac{\Gamma(m)}{\Gamma(m+0.5)}\right]^{2}-1}}
$$

Now, we will explore this distribution for various values of $m$ in the connection with physical conditions [7].

Consider the first the case, where large number of scatters is present within the resolution cell and there is no correlation between them. For this case $m=1$ 
and Nakagami distribution changes to Rayleigh distribution with one parameter $\Omega$ :

$$
f(S)=\frac{2 S}{\Omega} \cdot e^{-\frac{S^{2}}{\Omega}}
$$

where $E\left(S^{2}\right)=\Omega$ and represents reflected power. For this case the $S N R_{N}=$ 1.91 .

Consider now a case with presence of subresolvable periodic structure (we cannot distinguish between particulary scatters) with spacing $\lambda / 2$, where $\lambda$ corresponds to the wavelength and a collection of randomly located scatters. Under these condition the envelope will be Rician distributed with a density function

$$
f(S)=\frac{S}{\sigma^{2}} \cdot e^{-\frac{S^{2}+S_{0}^{2}}{2 \sigma^{2}}} I_{0}\left(\frac{S . S_{0}}{\sigma}\right)
$$

where $S_{0}$ represents a coherent component (the mean value of the inphase component arising from $\lambda / 2$ spacing) and $I_{0}($.$) is the zero-order modified Bessel$ function. $\sigma$ is a quantity describing random (diffuse) component [6]. The relation between the Nakagami parameter $m$ and Rician parameters is:

$$
\frac{1}{m}=1+\frac{S_{0}^{4}}{\left(2 \sigma^{2}+S_{0}^{2}\right)^{4}}
$$

The ratio $S N R_{R}=\frac{S_{0}}{\sigma}$ indicates the SNR. Thus the equation (10) can be rearranged to

$$
m=\frac{1+S N R_{R}^{2}+\frac{1}{4} S N R_{R}^{4}}{1+S N R_{R}^{2}} .
$$

As the $S N R_{R}$ increases the $m$ increases too (the subresolvable structure is more strong than diffuse scatters) because of the four power in the numerator. The $S N R_{N}$ will achieve values higher than 1.91 .

Consider another case of scattering structure - the spacing of periodic subresolvable structure is less than $\lambda / 4$. The pdf for this case is

$$
f(S)=\frac{S}{\sigma_{1} \sigma_{2}} \cdot e^{-\left(\frac{S^{2}}{4 \sigma_{1}^{2}}+\frac{S^{2}}{4 \sigma_{2}^{2}}\right)} I_{0}\left[\frac{S^{2}}{4}\left(\frac{1}{\sigma_{1}^{2}}-\frac{1}{\sigma_{2}^{2}}\right)\right]
$$

Signal-to-noise $S N R_{N}$ ratio for signal coming from this distribution is less than 1.91 (Rayleigh value). As the number of periodic located scatters becomes smaller the SNR approach to 1.91 and $m$ approach to 1 . The connection between the Nakagami and generalized Rician distribution is for values $0.5<m<1$ [7].

In some applications the value of $m$ is constrained such that $m \geq 0.5$. This corresponds to the half-Gaussian shape of the pdf (see Fig 1). However, the $m$-value can be less than 0.5 in ultrasound application [7. This situation corresponds to the very low scatter density. The shape of pdf is more sharp with heavy tail. Four pdf plots of the Nakagami distribution are shown on Fig[1. 

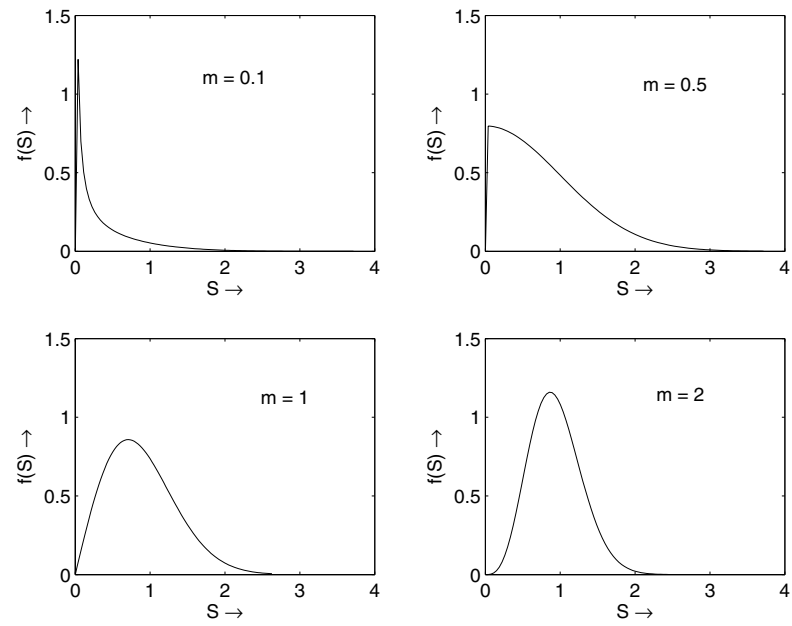

Fig. 1. Plots of the Nakagami pdf for different values of $\mathrm{m}$ and $\Omega=1$

\section{$4 \quad$ Model Based Filtering}

The idea for model-based filtering comes from the basic model for adaptive filter [1:

$$
y(i, j)=\bar{x}-k(i, j) \cdot(\bar{x}-x(i, j)),
$$

where $y(i, j)$ is a pixel of the output image, $\bar{x}$ is the mean of the window sample and $k(i, j)$ stands for adaptive factor that controls the filtering operation. Speckles are in fact an impulsive noise and it can be eliminated more effective by median filter rather than mean filter. Therefore the value $\bar{x}$ is replaced by median value. The key parameter of this filtering concept is to find $k(i, j)$, which is usually based on signal-to-noise ratio value. We try to design this parameter based on the $m$ parameter from the Nakagami pdf.

The filtering operation is performed on the non-interpolated envelope image. This means that we don't use an interpolated samples, but only original data (before scan conversion, data reduction and logarithmic compression) (see Fig 2). Let's go over the above mentioned cases for tissue. First, assume the low number of scatters such that $m<0.5$. This occurs in medium with very low density (e.g. blood) and also at the boundary with different acoustic impedances (specular reflections). In this case there should be no filtering, because of edge blurring. Therefore the $k$ should be adjusted to 1 . The second case is for $0.5<m<1$. The tissue has periodic unresolvable structure with spacing less than $\frac{\lambda}{2}$. The speckles are present and we should perform some kind of filtering. Therefore $k$ should be 1 for $m=0$ (no filtering) and for $m$ close to $1, k$ should be close to 0 . Situation for $m=1$ corresponds to Rayleigh (random) scattering and $k=0$ (maximum filtering). 


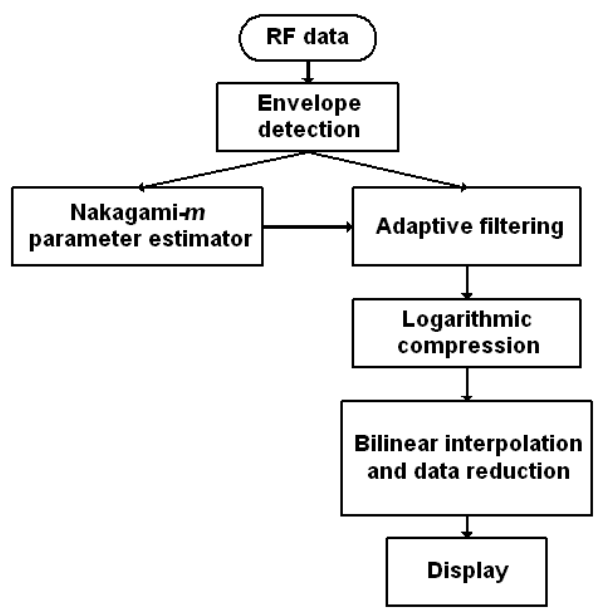

Fig. 2. Algorithm for adaptive filtering using Nakagami parameter

Table 1. Relation between $\mathrm{m}$ and $\mathrm{k}$ parameter

\begin{tabular}{|c|c|c|}
\hline Nakagami-m & Tissue & Parameter $\mathrm{k}$ \\
\hline$m<0.5$ & $\begin{array}{c}\text { low scatters density or } \\
\text { specular reflection }\end{array}$ & $k=1$ \\
\hline $0.5 \leq m<1$ & $\begin{array}{c}\text { unresolvable structure } \\
\text { with spacing }<\frac{\lambda}{2}\end{array}$ & $\begin{array}{c}k=1 \text { for } m=0.5 \text { and } \\
\text { k close to } 0 \text { for } m \text { close to } 1\end{array}$ \\
\hline$m=1$ & random scattering & $k=1$ \\
\hline$m>1$ & $\begin{array}{c}\text { unresolvable structure } \\
\text { with spacing } \frac{\lambda}{2}\end{array}$ & k increase with increasing $m$ \\
\hline
\end{tabular}

The last situation is for tissues with subresolvable periodic structure with spacing $\lambda / 2$. As the ratio of this structure to random scatters rises, the $m$ rises too. Therefore the filter must operate such that $k$ is close to 0 for $m$ close to 1 and with increasing value of $m$ (and increasing SNR) the $k$ should fall to 0 (minimum filtering). These conditions are summarized in Tab1.

The quality of estimator for the $m$-parameter is an important part for Nakagami based filter. More details can be found in [5], where the comparison of several estimator is made. We've used an approximating estimator as proposed by Greenwood and Durand in 1960.

Next problem arises with the size of the window. There are in fact two windows: one for $m$ parameter estimation and the second window for filtering. We use the same window size for both cases. For too small window we obtain only few samples to estimate the parameter $m$. For the large window we decrease the spatial resolution. The choice is trade-off between this two negative properties. It seems reasonable to not to decrease the lateral resolution, because in nowadays ultrasound scanners this resolution is poor in comparison with the 
axial resolution. Therefore the minimum window size in lateral direction is 3 (an odd number for simple computing). The resolution in axial direction is better and corresponds to the wavelength and central frequency of the ultrasound wave $\lambda=\frac{c}{f}$. Assuming $c=1540 \mathrm{~m} . \mathrm{s}^{-1}$ and $f_{0}=2 \mathrm{MHz}$ the wavelength is $0.77 \mathrm{~mm}$ and the resolution can be considered as a $\lambda / 2$. Axial resolution also depends on the pulse length (number of cycles), which is usually about 4 . The corresponding number of samples depends on the sampling frequency $\left(f_{s}=20 \mathrm{MHz}\right.$ in our case) and is given by $n=\frac{4}{2} \frac{f_{0}}{f_{s}}=20$. The window size should be higher than this value because of obtaining representative sample to determine the $m$ parameter. Several window sizes were tested and as an appropriate size is between 19 and 35 samples. This match with the length from $1.33 \mathrm{~mm}$ to $2.67 \mathrm{~mm}$.

\section{$5 \quad$ Filtering Results and Discussion}

The filtering performance was tested on the simulated images with different scatterers densities. A one-dimensional discrete scattering model was used in our simulation [5]. Fig. 3a) shows simulated ultrasound image with random scatter density 2, 5, 20 within resolution cell. Fig. 3b) shows filtering result after applying the proposed method( Nakagami filter ). One can see that the smoothing depends on the scatter densities. For low scatter density (upper part of the image) there is almost no smoothing. Middle and lower part of the image

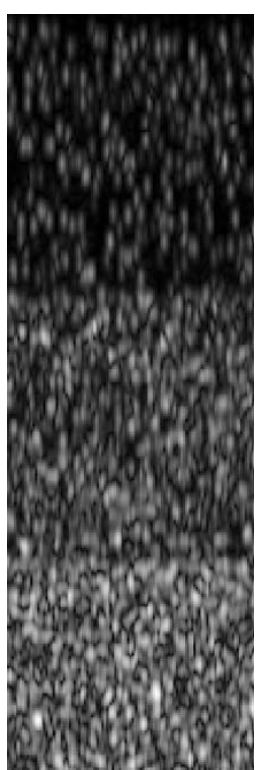

a)

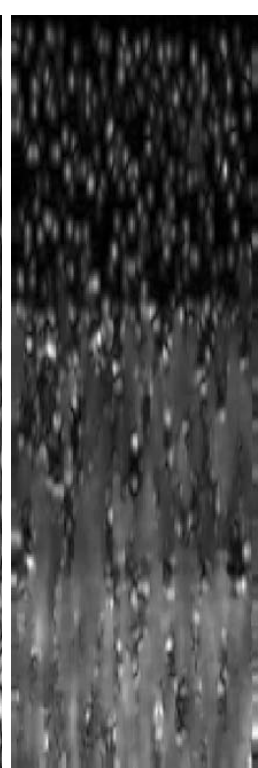

b)

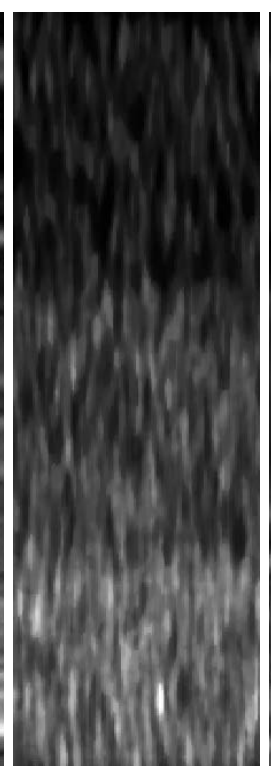

c)

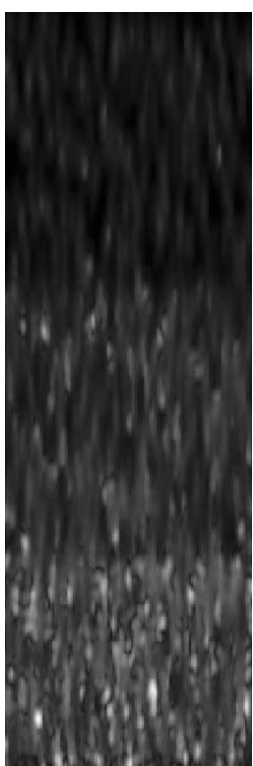

d)

Fig. 3. Simulated image with different scatter densities: a) Original image , Filtered image by b) the proposed method, c) median filter, d) Wiener filter 
Table 2. The SI Values for Simulated Image

\begin{tabular}{|l|c|c|c|}
\hline region & $\mathbf{1}$ & $\mathbf{2}$ & $\mathbf{3}$ \\
\hline \hline original & 0.91 & 1.61 & 1.84 \\
\hline Nakagami filter & 0.96 & 2.94 & 3.45 \\
\hline Median filter & 1.30 & 4.1 & 4.2 \\
\hline Wiener filter & 1.9 & 3.80 & 3.10 \\
\hline
\end{tabular}

are more smoothed. If some stronger reflection is presence it can be seen that the smoothing performance is decreased. The proposed method was compared with the simple median filter and adaptive Wiener filter as implemented in Matlab. This Wiener filter estimates the local mean and variance around each pixel within the sliding window and compute the estimate of new sample 9. The speckles can be quantified by the means of speckle index (SI) evaluated for homogenous region [2]:

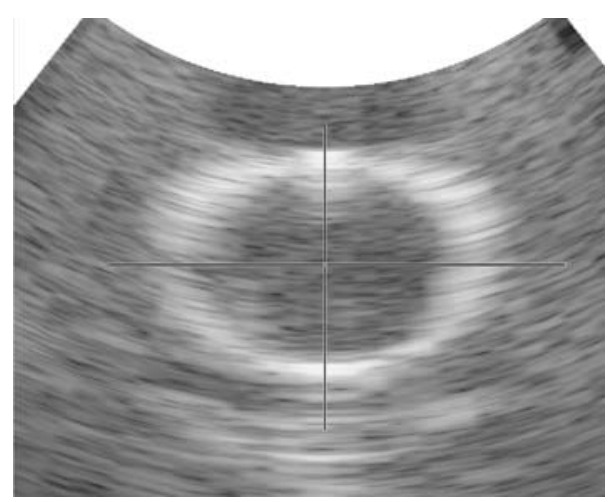

a)

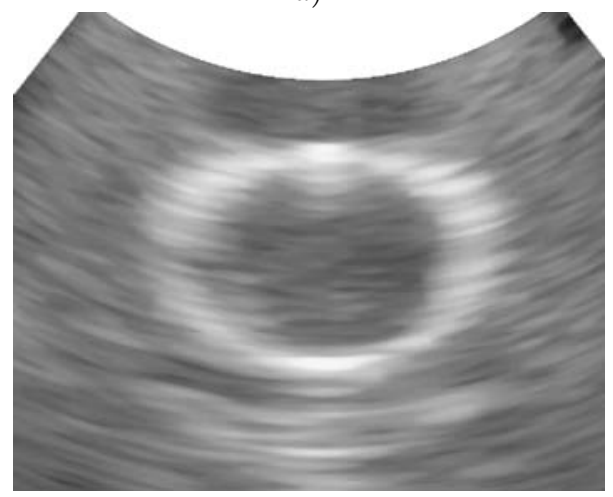

c)

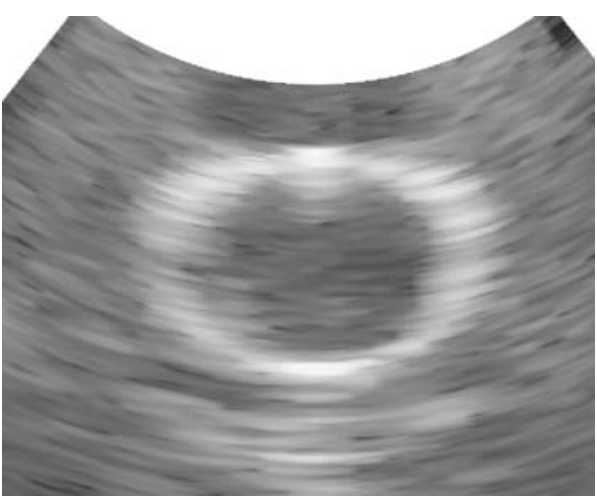

b)

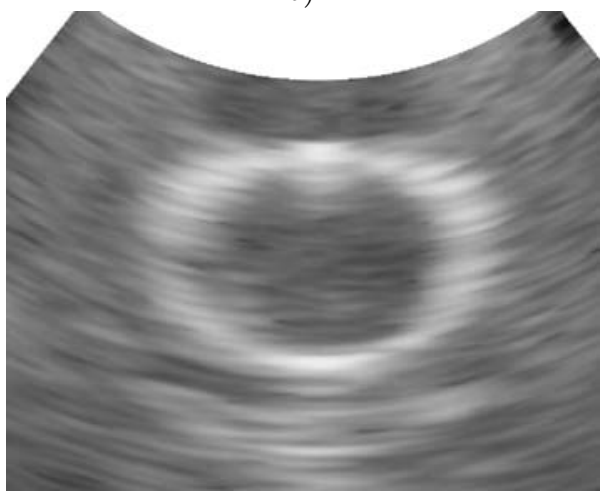

d)

Fig. 4. Real images of circular shaped object: a) Original image , b) After Nakagami filtering, c) median filtering and d) Wiener filtering 


$$
S I=\frac{\mu}{\sigma}
$$

where $\mu$ and $\sigma$ are region mean value and standard deviation. From the view of SI evaluation it seems that the median filter is the best choice for speckle suppression. This is can be true for homogeneous regions, because the speckles are consider as an impulsive noise. On the other hand, real tissues are highly nonhomogeneous and some advanced method should be employed for speckle filtering.

Sample of circular object with strong reflecting borders is shown on Fig 4 a) and the image after filtering by Nakagami filter (window size $31 \times 3$ ) is shown on Fig 4 b). Fig 4 c,d) shows the images after median and Wiener filtering with the

a)

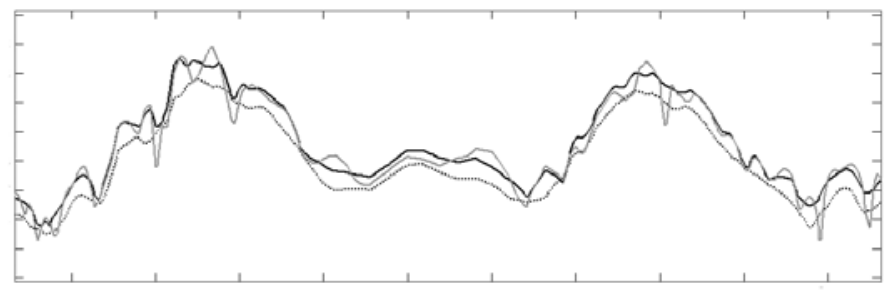

b)

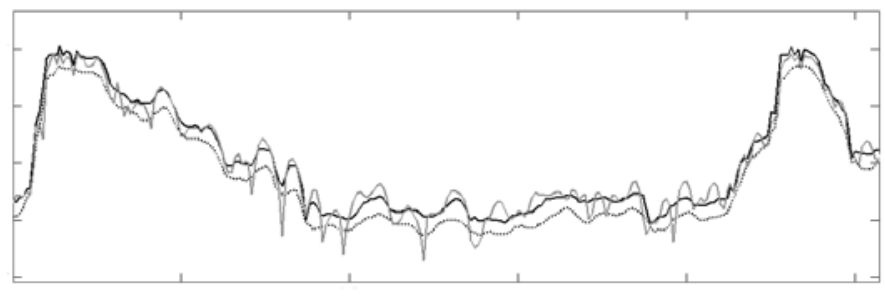

Fig. 5. 1D profile of the circular object. Original profile (gray line), Nakagami filtered (black line), median filtered (dotted line)

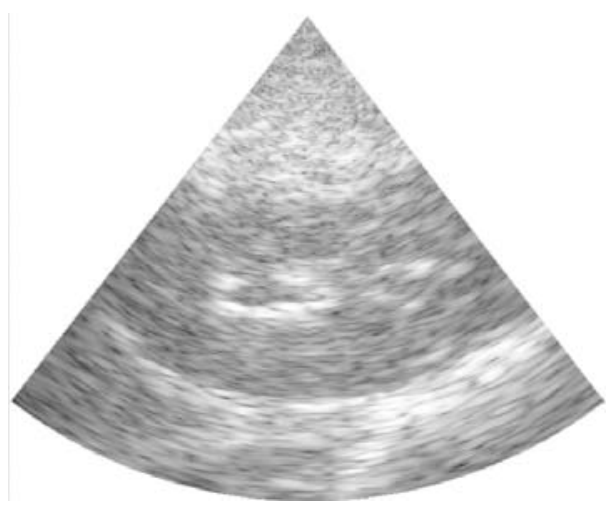

a)

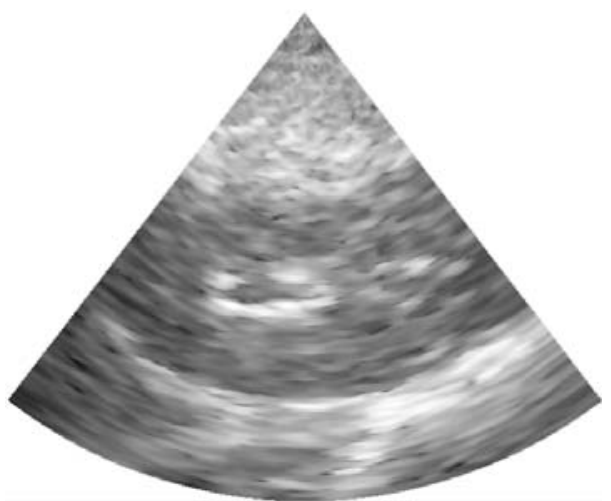

b)

Fig. 6. Real images of the kidney: a) Original image , b) Nakagami filtered image 
same size of window. The filtering was made on the envelope of the RF matrix before scan conversion. The blurring artefact is more obvious in images c) and d), particulary in lateral direction. After Nakagami filter the edges remains more sharp and the speckles are reduced. This is shown on Fig 5, where the vertical and horizontal cross-sections through this circular object are depicted. Only Wiener filtered image is shown on Fig 5. The 1D profile for median filter is very similar and therefore is not shown. Real tissue Nakagami filtering was also performed. The kidney and heart was tested and visually evaluated. Samples of kidney filtering is shown on Fig 6 . The probe frequency was $3.3 \mathrm{MHz}$, depth of scanning $10 \mathrm{~cm}$.

\section{Conclusion}

The new method for speckle reduction in ultrasound images has been presented. It uses the parameter $m$ from the Nakagami distribution and it therefore depends on the quality of estimators. This problem hasn't been discussed here in details. We've focused only on the design of the adaptive filter. The design is in fact ad $h o c$ and empirical for the intention of speckle elimination. The apparent speckle reduction was achieved both, in simulated and in ultrasound images from phantom and real tissues, while the edges remain sharp.

\section{Acknowledgment}

This work has been supported by the grant of Ministry of Education (Czech republic) CEZ MS 0021630513 and the grant no. 102/03/P153 of Czech Science Foundation.

\section{References}

1. Bamber J.C., Daft C., Adaptive filtering for reduction of speckle in ultrasonic puls-echo images, Ultrasonics, January, 1986, pp. 41-44.

2. Crimmins,T.R., Geometric filter for speckle reduction, Applied Optics vol.24, no.10, pp.1438-1443, 1985

3. Dutt, V. Statistical Analysis of Ultrasound Echo Envelope, PhD Thesis, The Mayo Graduate Scholl, 1995

4. Insana,M.F. et al. On the Information Content of Diagnostic Ultrasound, Proceedings of the Tenth International Conference on Information Processing in Medical Imaging, 1987, pp.437-455

5. Kolář R., Jiřík R., Jan J. Estimator Comparison of the Nakagami-m Parameter and its Application in Echocardiography, Radioengineering, April, 2004, vol. 13, no. 1 , pp.8-12

6. Kolář, R., Kozumplík, J.: Noise Suppresion in Ultrasound Images Using Wavelets. Proceedings of the International Conference Applied Electronics 2001, Sept. 2001, Pilsen, pp. 130-133. 
7. Shankar, P.M. Ultrasonic Tissue Characterization Using a Generalized Nakagami Model, IEEE Trans. on Ultrasonics, Ferroelectrics and Frequency Control, 2001, vol. 48 , no. 6 , pp. 1716-1720.

8. Shankar, P.M., A General Statistical Model for Ultrasonic Backscattering from Tissues, IEEE Trans. on Ultrasonics, Ferroelectrics and Frequency Control, 2000, vol. 47 , no. 3, p. 727-736.

9. User's Guide, Image Processing Toolbox, Mathworks, http://www.mathworks.com

10. Zhang, Q.T., A Note on the Estimation of Nakagami-m Fading Parameter, IEEE Communications Letters, 2002, vol. 6, no. 6, p. 237-238. 